\title{
Voluntary Modulation of Evoked Responses Generated by Epidural and Transcutaneous Spinal Stimulation in Humans with Spinal Cord Injury
}

\author{
Jonathan S. Calvert ${ }^{1}{ }^{\oplus}$, Megan L. Gill ${ }^{2}$, Margaux B. Linde ${ }^{2}$, Daniel D. Veith ${ }^{2}$, Andrew R. Thoreson ${ }^{2}$, \\ Cesar Lopez ${ }^{2}$, Kendall H. Lee ${ }^{2,3,4}$, Yury P. Gerasimenko ${ }^{5,6}$, Victor R. Edgerton 7,8,9,10,11,12@ , Igor A. Lavrov ${ }^{3}$, \\ Kristin D. Zhao ${ }^{2,4}$, Peter J. Grahn ${ }^{2,3}$ and Dimitry G. Sayenko ${ }^{13, *}$
}

check for updates

Citation: Calvert, J.S.; Gill, M.L.; Linde, M.B.; Veith, D.D.; Thoreson, A.R.; Lopez, C.; Lee, K.H.; Gerasimenko, Y.P.; Edgerton, V.R.; Lavrov, I.A.; et al. Voluntary Modulation of Evoked Responses Generated by Epidural and Transcutaneous Spinal Stimulation in Humans with Spinal Cord Injury. J. Clin. Med. 2021, 10, 4898. https:// doi.org/10.3390/jcm10214898

Academic Editors: Ursula S. Hofstoetter and Karen Minassian

Received: 12 September 2021 Accepted: 20 October 2021 Published: 24 October 2021

Publisher's Note: MDPI stays neutral with regard to jurisdictional claims in published maps and institutional affiliations.

Copyright: (c) 2021 by the authors. Licensee MDPI, Basel, Switzerland. This article is an open access article distributed under the terms and conditions of the Creative Commons Attribution (CC BY) license (https:// creativecommons.org/licenses/by/ $4.0 /)$.
1 Mayo Clinic Graduate School of Biomedical Sciences, Mayo Clinic, Rochester, MN 55905, USA; jonathan_calvert@brown.edu

2 Department of Physical Medicine and Rehabilitation, Mayo Clinic, Rochester, MN 55905, USA; gill.megan@mayo.edu (M.L.G.); linde.margaux@mayo.edu (M.B.L.); veith.daniel@mayo.edu (D.D.V.); thoreson.andrew@mayo.edu (A.R.T.); lopez.cesar@mayo.edu (C.L.); lee.kendall@mayo.edu (K.H.L.); zhao.kristin@mayo.edu (K.D.Z.); grahn.peter@mayo.edu (P.J.G.)

3 Department of Neurologic Surgery, Mayo Clinic, Rochester, MN 55905, USA; lavrov.igor@mayo.edu

4 Department of Physiology and Biomedical Engineering, Rochester, MN 55905, USA

5 Pavlov Institute of Physiology of Russian Academy of Sciences, 199034 St. Petersburg, Russia; yury.gerasimenko@louisville.edu

6 Department of Physiology and Biophysics, University of Louisville, Louisville, KY 40292, USA

7 Department of Integrative Biology and Physiology, University of California Los Angeles, Los Angeles, CA 90095, USA; vre@ucla.edu

8 Department of Neurobiology, University of California Los Angeles, Los Angeles, CA 90095, USA

9 Department of Neurosurgery, University of California Los Angeles, Los Angeles, CA 90095, USA

10 Brain Research Institute, University of California Los Angeles, Los Angeles, CA 90095, USA

11 Institut Guttmann, Hospital de Neurorehabilitació, Institut Universitari Adscrit a la Universitat Autònoma de Barcelona, 08916 Badalona, Spain

12 Centre for Neuroscience and Regenerative Medicine, Faculty of Science, University of Technology Sydney, Ultimo 2007, Australia

13 Center for Neuroregeneration, Department of Neurosurgery, Houston Methodist Research Institute, Houston, TX 77030, USA

* Correspondence: dgsayenko@houstonmethodist.org; Tel.: +1-713-363-7949 
Keywords: spinal cord injury; electrically evoked spinal motor potentials; spinal cord stimulation; neuromodulation

\section{Introduction}

Transcutaneous (TSS) and epidural spinal stimulation (ESS) are electrical neuromodulation approaches that have previously been used to modulate spinal sensorimotor networks in humans [1,2]. Both TSS and ESS have been shown to enable motor functions previously thought to be permanently lost in individuals with paraplegia due to spinal cord injury (SCI), such as voluntary movement of previously paralyzed limbs [3-8], standing [9-12], and stepping [13-15]. TSS and ESS are both hypothesized to increase the level of excitability below the injury level, allowing previously silent, intact neural tissue that remains following injury to access sensorimotor networks responsible for function below the injury $[16,17]$. TSS and ESS have been shown to recruit common neural structures in electrophysiological [18] and computational modeling studies [19,20]. However, the ability of individuals with SCI to modulate epidural and transcutaneous spinally evoked motor potentials has not been investigated in detail (Figure 1A).
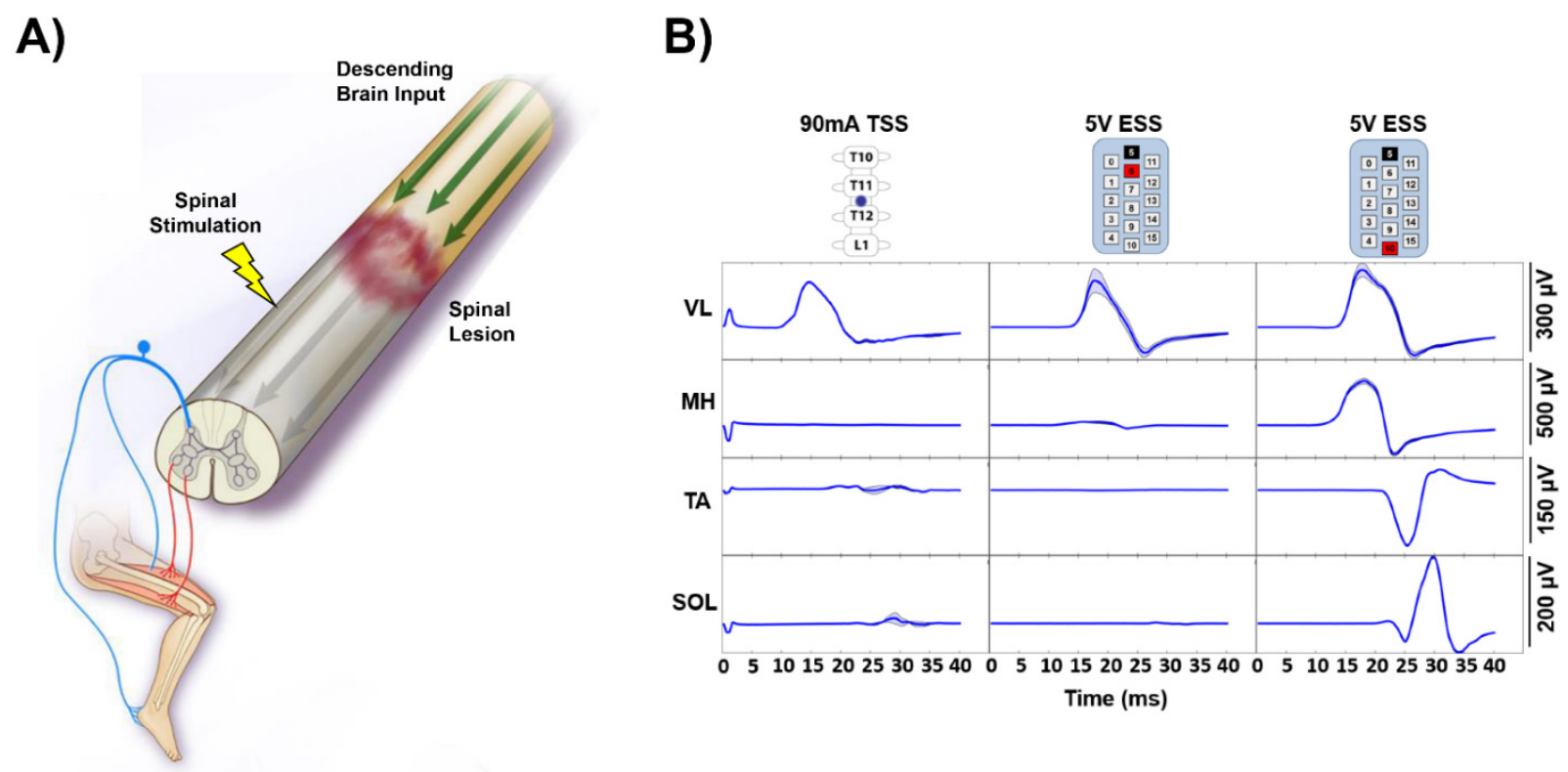

Figure 1. TSS- and ESS-Evoked Responses While Relaxed. (A). A diagram depicting inputs and outputs to the spinal cord during spinal stimulation. Descending brain input (green arrows) is interrupted by the spinal cord lesion. Spinal stimulation (yellow lightning bolt) is hypothesized to function by activating the dorsal roots carrying afferent proprioceptive information to the spinal cord. Afferent proprioceptive inputs (blue) enter the spinal cord and efferent motor outputs (red) exit the spinal cord and returns to the muscle. This figure is adapted with permission from a previous publication [6]. (B). While a study participant was instructed to relax while lying supine, stimulation was delivered to the same region of the spinal cord via transcutaneous spinal stimulation (TSS) and epidural spinal stimulation (ESS) using a focal and wide field. The dark line represents the average of at least three stimuli, and the shaded region indicates the \pm standard deviation. VL—vastus lateralis, $\mathrm{MH}$-medial hamstrings, $\mathrm{TA}$ - tibialis anterior, SOL—soleus, $\mu \mathrm{V}$-microvolt, $\mathrm{V}$ —Volt, and $\mathrm{mA}-$ milliamp.

Previous reports of ESS and TSS have investigated spinally evoked responses via electromyography (EMG) of upper [21,22] and lower-extremity $[4,23,24]$ musculature to characterize the effect of electrode location, different stimulation parameters, and body position on the motor thresholds and gain properties of sensorimotor networks. In these studies, stimulation was applied at low frequency ranges $(0.2-2 \mathrm{~Hz})$ in order to evaluate sensorimotor output while minimizing the effects of post-activation depression from 
frequent stimulation [25]. Previous reports indicate that some study participants, clinically diagnosed as having a motor complete SCI, were able to show signs of a non-specific, generalized increase in EMG activity below their injury level when asked to perform a full body muscle contraction by maximally flexing the muscles rostral to the SCI $[7,13,26]$. This has brought renewed focus to discomplete injuries, where study participants demonstrate motor activity via EMG in specific reinforcement tasks, despite being clinically classified in the ASIA (American Spinal Injury Association) Impairment Scale (AIS) as having a motor complete SCI $[26,27]$. Study participants without a SCI have demonstrated increased, as well as decreased, amplitude of TSS-evoked responses in some muscles during voluntary tasks $[21,28,29]$. However, the effect of voluntary control in individuals with SCI over TSSor ESS-evoked responses has yet to be examined.

Here, we investigated the effect of voluntary control on TSS- and ESS-evoked responses in individuals with SCI at a range of injury severities. Participants were tested in two different conditions while supine: relaxed and while attempting maximal voluntary flexion of the lower extremities. During these tasks, spinally evoked motor potentials were recorded via EMG from the lower extremities. As previous work has demonstrated that individuals with SCI can increase the amplitude of EMG recordings taken from below the SCI, we hypothesized that voluntary attempts would increase spinally evoked response amplitude when compared to the relaxed condition.

\section{Methods}

\subsection{Description of Participants}

The experimental procedures described herein were approved by the respective University of California, Los Angeles (UCLA) and Mayo Clinic institutional review boards, and study participants provided written, informed consent to the experimental procedures. Data from two independent investigations were retrospectively analyzed via collaborative efforts from investigators at both institutions. Experiments were conducted in nine participants (seven at UCLA, two at Mayo Clinic) with chronic SCI (see Table 1 for full demographics). Study participants sustained an SCI at least two years prior to study enrollment. Two study participants were part of a study at the Mayo Clinic whose functional motor responses have previously been reported $[4,7,13,30,31]$. These publications focused on motor outputs during functional tasks such as voluntary control of lower-extremity muscles, stepping, standing, and sitting [7,13,30,31], as well as intraoperative recordings [4]. All data and analyses from these participants in this report were recorded at low $(0.2-2 \mathrm{~Hz})$ non-functional stimulation frequencies while the subjects were supine. All data contained within this manuscript have not previously been published. Briefly, these study participants performed six months of task-specific training, including body weight supported treadmill and over ground training without stimulation. At the initiation and conclusion of these six months, TSS was applied at the T10-L1 spinal vertebral levels to assess the sensorimotor connectivity of the lower-extremity musculature and spinally evoked motor responses prior to implantation of the epidural stimulator. Following these six months, participants were implanted with an epidural stimulator (Specify 5-6-5, Medtronic, Fridley, MN, USA) [4] and performed 12 months of multi-modal rehabilitation which paired taskspecific rehabilitation with ESS [7]. The other seven participants were part of a study on the effects of TSS on trunk stability and self-assisted standing at the University of California, Los Angeles [12,32]. However, all data and analysis in this report are unpublished, and the study participants did not receive spinal stimulation prior to study enrollment. 
Table 1. Study Participant Demographics.

\begin{tabular}{ccccccc}
\hline Subject ID & Sex & Age & Injury Level & Time Since Injury & AIS Score & Stimulation Modality \\
\hline N01 & Male & 26 & T6 & 3 years & A & ESS, TSS \\
N02 & Male & 36 & T3 & 6 years & ESS, TSS \\
N03 & Male & 22 & C5 & 5 years & B & TSS \\
N04 & Male & 26 & T2 & 8 years & A & TSS \\
N05 & Female & 32 & C5 & 13 years & A & TSS \\
N06 & Male & 23 & T2 & 7 years & A & TSS \\
N07 & Male & 25 & T4 & 7 years & C & TSS \\
N08 & Male & 26 & C4 & 2 years & C & TSS \\
N09 & Male & 28 & T4 &
\end{tabular}

This table depicts the demographics of the study participants including their study ID, sex, age, injury level, time since injury, AIS (American Spinal Injury Association Impairment Score), and stimulation modality. ESS-epidural spinal stimulation; TSS-transcutaneous spinal stimulation.

\subsection{Data Acquisition}

Surface electromyogram (EMG) signals were recorded using bipolar self-adhesive electrodes placed longitudinally over the muscle belly of the vastus lateralis (VL), medial hamstrings (MH), tibialis anterior (TA), and soleus (SOL) muscles of each leg. Signals were differentially amplified and digitized at a sampling rate of 4000 samples per second (PowerLab, ADInstruments, Dunedin, New Zealand) and stored electronically (LabChart, ADInstruments, Dunedin, New Zealand). EMG data were analyzed offline using custom code written in MATLAB (Version R2020a, The Mathworks Inc., Natick, MA, USA) following application of a notch filter at $60 \mathrm{~Hz}$ and a 2nd order bandpass filter between 10 and $1000 \mathrm{~Hz}$. All EMG recordings were synchronized to each pulse of TSS or ESS via stimulus artifact recorded from an electrode placed on the surface of the thoracolumbar spine.

Study participants were instructed to perform two experimental tasks with and without spinal stimulation: (1) to stay relaxed while lying supine to establish a control condition, and (2) to put forth maximum effort in attempting a single leg flexion maneuver including hip flexion, knee flexion, and ankle dorsiflexion simultaneously. A subset of subjects was also asked to perform joint-specific movements (e.g., plantarflexion, dorsiflexion) in the presence of stimulation. Each task was performed for at least three trials in each leg by each participant. During voluntary tasks, stimulation was delivered at a global motor threshold, which was defined as the stimulation amplitude where the peak-to-peak amplitude of all recorded muscles exceeded $20 \mu \mathrm{V}$ responses.

\subsection{Stimulation Procedures}

Transcutaneous spinal stimulation was delivered either using a DS7A Biphasic Constant Current Stimulator (Digitimer, Hertfordshire, UK) or a custom-built, three channel constant-current stimulator. Stimulation was administered via self-adhesive electrodes (PALS, Axelgaard Manufacturing Co., Ltd., Fallbrook, CA, USA) with a diameter of $3.2 \mathrm{~cm}$ placed on the skin at the spinal midline between spinous processes from the T11 to L2 vertebrae to act as cathodes. Two $5 \mathrm{~cm} \times 10 \mathrm{~cm}$ self-adhesive electrodes (PALS, Axelgaard Manufacturing Co., Ltd., Fallbrook, CA, USA) were placed symmetrically on the skin longitudinally over the abdomen for use as anodes. During TSS, stimuli were delivered as monophasic rectangular pulses with a $1 \mathrm{~ms}$ pulse width. Stimuli were delivered at 0-150 $\mathrm{mA}$ at stimulation frequencies between 0.2 and $2 \mathrm{~Hz}$. A minimum of three stimuli were delivered during each trial.

Epidural spinal stimulation (ESS) was delivered using an implantable spinal cord stimulator (Specify 5-6-5, Medtronic, Fridley, MN, USA) placed between the T11-L1 vertebral bodies connected to an implanted pulse generator (RestoreSensor Sure-Scan MRI, Medtronic, Fridley, MN, USA). During ESS, stimuli were delivered as biphasic chargebalanced rectangular pulses with a $0.21 \mathrm{~ms}$ pulse width at a frequency of $0.2-2 \mathrm{~Hz}$. Each electrode could be configured as a cathode, anode, or off. The electrode configurations were defined empirically based on the motor outputs of each subject, and were used to target 
specific rostral-caudal locations of the spinal cord that would enable either specific motor activation of proximal or distal lower-extremity musculature, or non-specific activation of multiple muscles of the lower extremity. ESS-evoked motor response recordings were captured during multiple ESS configurations and stimulation parameters with wide or local current distributions at the rostral and caudal ends of the electrode array $(0-10 \mathrm{~V})$.

\subsection{Data Processing and Statistics}

Mean and standard deviation values were calculated from at least 3 consecutive stimuli. Magnitudes of the spinally evoked potentials were calculated by measuring the area under the curve by applying a trapezoidal numerical integration to rectified EMG signals from 5 to $45 \mathrm{~ms}$ after the stimulus to capture the entire evoked response and prevent stimulation artifact contaminating the EMG signal. The evoked responses during voluntary contraction were normalized to the response in each muscle during the relaxed condition to account for individual differences during EMG collection in each participant. Statistically significant differences across the entire population of subjects were determined using the Wilcoxon signed-rank test for all EMG data $(p<0.05)$ using the signrank function in MATLAB, as the data were not normally distributed. The data used for the statistical tests were calculated by taking the average normalized area under the curve value of the first three evoked responses for each of the 9 subjects within the study population. After the average value was obtained for each participant, these data were entered into the signrank function to calculate the $p$-values for each recorded muscle. The paired, two-sided Wilcoxon signed-rank test was chosen over the Wilcoxon rank-sum test, as the data were from matched samples. However, comparisons across population subgroups did not have a large enough sample size to confirm statistical significance. Raw and processed datasets are available from the corresponding author upon request.

\section{Results}

\subsection{Epidural and Transcutaneous Spinal Stimulation in the Same Participants}

When stimulation was delivered at similar intensities at different electrode configurations, TSS applied at the T11/T12 intervertebral location and ESS applied at a focal, rostral portion of the electrode array $(-5 /+6)$ resulted in distinct evoked responses in the VL with relatively little activation in the other recorded muscles (MH, TA, SOL) (Figure 1B). When ESS was set with a wide field configuration $(-5 /+10)$ at the same stimulation intensity, all recorded muscles (VL, MH, TA, SOL) were activated.

\subsection{Effect of Voluntary Effort on Spinally Evoked Responses}

As shown in a representative ESS study participant and a representative TSS study participant, stimulation at motor threshold resulted in evoked responses in the leg muscles while the participants were relaxed (Figure 2A). However, when the participants were instructed to perform a full leg flexion, lower-extremity muscle responses were decreased compared to the relaxed condition. The data were normalized to compare across all participants, and the average area under the curve of the first three evoked responses was calculated for each of the nine study participants. When compared across the entire study population, the average area under the curve of the evoked responses was significantly lower across all recorded EMG muscles during the voluntary attempts to perform the leg flexion compared to the relaxed condition (mean \pm standard error, $p$-value; VL: $0.6801 \pm$ $0.1110, p=0.0117$; MH: 0.7084 $\pm 0.1157, p=0.0391$; TA: $0.6208 \pm 0.1327, p=0.0391$; SOL: $0.4545 \pm 0.1048, p=0.0039$ ) (Figure 2B). Furthermore, a representative subject who was asked to perform joint-specific movements (i.e., plantarflexion and dorsiflexion) demonstrated inhibition of the evoked potentials across muscles on both sides of the body during both plantarflexion and dorsiflexion (Figure 3). 
A)

B)
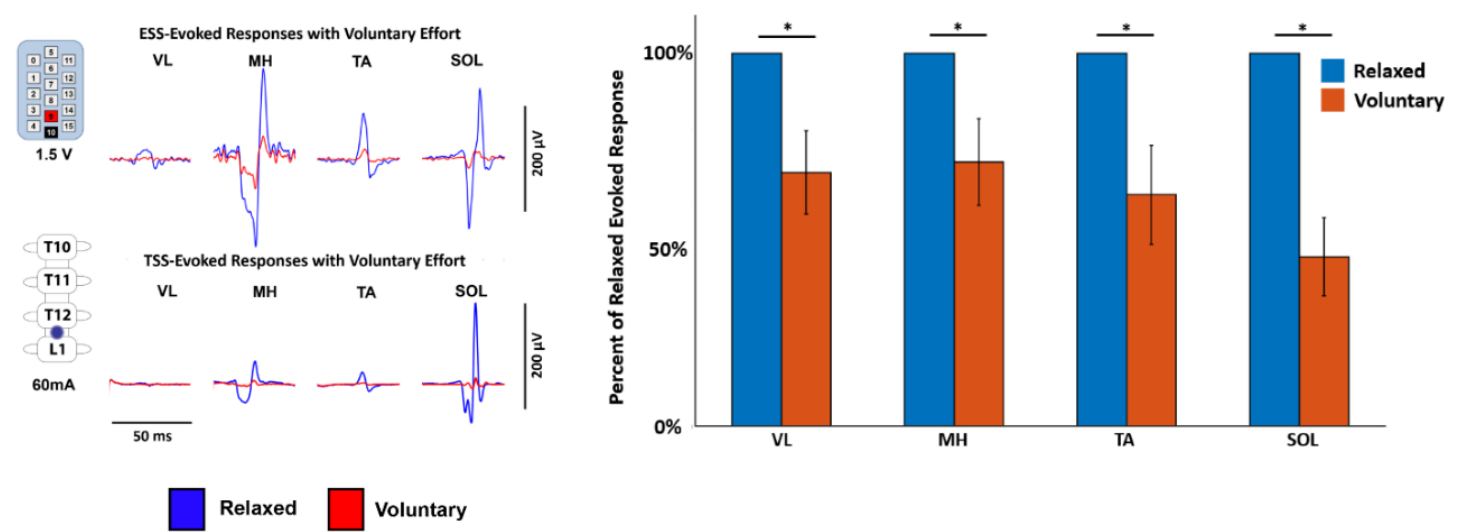

Figure 2. Inhibition of Evoked Response Amplitude During Voluntary Flexion. (A). Data from a representative study participant using ESS and a representative study participant using TSS while relaxed and while attempting maximum voluntary flexion of the lower extremities, which results in a decreased evoked response. Stimulation is delivered at the beginning of each trace. Blue indicates the relaxed condition and red indicates the voluntary flexion condition. (B). Grouped data from all participants within this study indicating significant decreases across all four recorded muscles when the voluntary flexion condition is compared to the relaxed condition. Data are normalized to the maximum EMG response in each muscle in each participant to compare across participants. Error bars represent the mean \pm standard error. $\mathrm{VL}$-vastus lateralis, $\mathrm{MH}$-medial hamstrings, TA—tibialis anterior, SOL—soleus, $\mu \mathrm{V}$-microvolt, $\mathrm{V}-\mathrm{Volt}$, $\mathrm{mA}$-milliamp, and $*<0.05$.

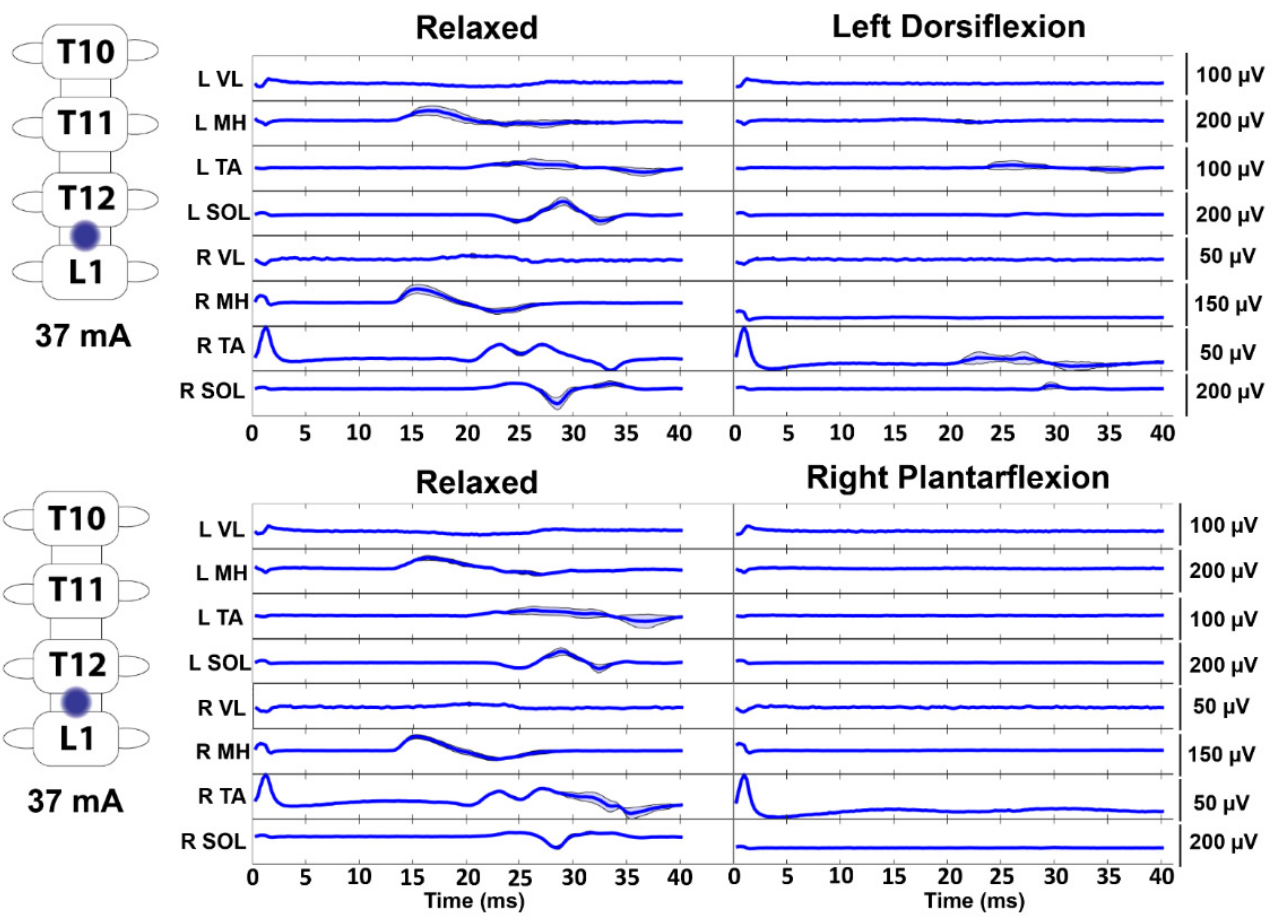

Figure 3. Joint-Specific Movements Decrease Motor-Evoked Responses. During T12/L1 stimulation in a representative study participant, motor-evoked responses were decreased across both the left and right lower extremities during attempts to voluntarily flex the ankle. Stimulation is delivered at the beginning of each trace. The dark line represents the average of at least three stimuli, and the shaded region indicates the \pm standard deviation. VL-vastus lateralis, $\mathrm{MH}-$ medial hamstrings, TA—-tibialis anterior, SOL—soleus, $\mu \mathrm{V}$-microvolt, $\mathrm{V}$-Volt, and $\mathrm{mA}$-milliamp. 


\subsection{Effect of Stimulation Modality and Injury Severity on Voluntary Modulation of Evoked Responses}

To examine if stimulation modality and injury severity had an effect on the ability to modulate the evoked responses, study participants were stratified into three groups: ESS with participants diagnosed with an AIS-A SCI, TSS with AIS-A SCI, and TSS with AIS-B/C $\mathrm{SCI}$. When the evoked responses were averaged across the entire voluntary contraction, both participants with AIS-A tested with ESS decreased the amplitude of their evoked responses when instructed to perform a full leg flexion (Figure 4). All participants tested with TSS were exposed to stimulation with the cathode positioned between the T12-L1 vertebral bodies. Both ESS participants used a symmetric 9+/10- configuration. In all three AIS-A participants tested with TSS, the amplitude of the evoked responses in at least 3 out of 4 of the recorded muscles did not fall outside of the standard deviation of the normalized relaxed value. However, all four AIS-B/C participants tested with TSS demonstrated a reduction in the evoked responses amplitude compared to the normalized relaxed value in at least 3 out of 4 of the recorded muscles. However, statistical comparisons across subgroups could not be made due to the low number of study participants in each subgroup.

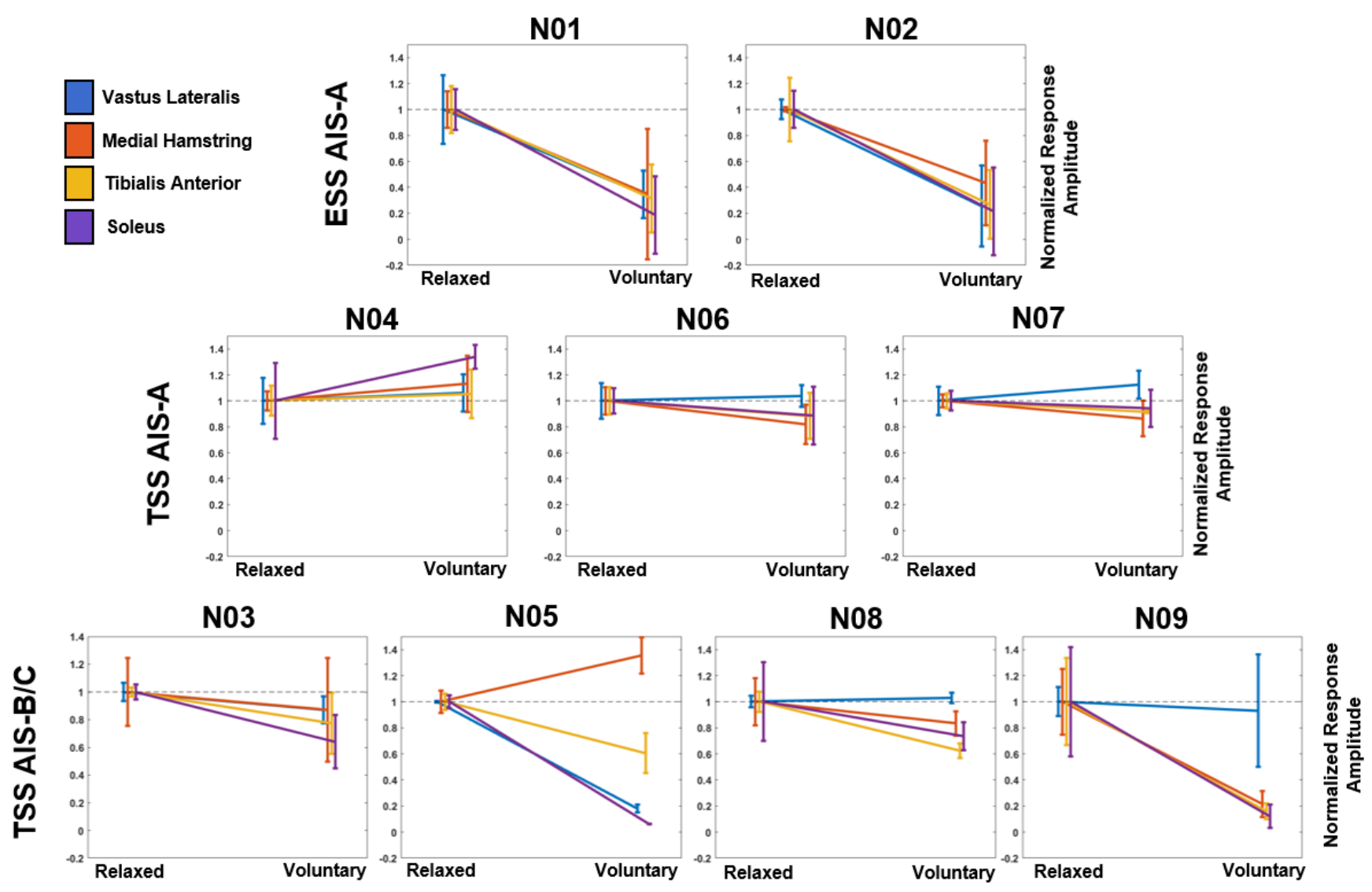

Figure 4. Evoked Response Modulation by ASIA Impairment Score. The first row indicates the two participants with clinically complete SCI tested with ESS. The second row indicates the three participants with clinically complete SCI tested with TSS. The third row indicates the four participants with clinically incomplete SCI tested with TSS. Data on the left of each plot refer to the average evoked response during the relaxed condition, and the data on the right refers to the average evoked response during the voluntary flexion condition. Data are normalized to the maximum EMG response in each muscle in each participant to compare across participants. The black dashed line indicates the average response of each muscle during the relaxed condition. Error bars represent the mean \pm standard deviation. AIS-American Spinal Injury Association Impairment Score, VL—vastus lateralis (blue), $\mathrm{MH}$-medial hamstrings (orange), TA—tibialis anterior (yellow), and SOL—soleus (purple). 


\section{Discussion}

ESS and TSS have demonstrated improvements across a wide range of functions in individuals with SCI $[3,4,6,9,11-15,32,33]$. However, the complex interactions between stimulus pulses, the descending commands originating above the SCI and passing through the lesion site, and afferent inputs during movements to produce the functional spinal sensorimotor network outputs remain poorly understood. Here, we demonstrate the inhibition of evoked responses from ESS and TSS during voluntary attempts of individuals with severe SCI to move paralyzed limbs while lying supine.

In study participants who were stimulated with both TSS and ESS, similar evoked muscle responses were observed when the subjects were instructed to relax (Figure 1). ESS and TSS have previously been shown to activate common neural structures in electrophysiological studies [18]. Furthermore, ESS and TSS have both been shown to preferentially activate rostral-caudal and medio-lateral spinal motor pools [34-37], and both modalities are proposed to function, in part, through activation of dorsal roots entering the spinal cord $[19,20,38]$. However, it remains unknown what degree of specificity in activation of particular motor pools is necessary to achieve a given level of functional restoration of movement. It can be reasoned that either a specific or a broad activation pattern may be useful in engaging sensorimotor circuitry necessary for different functional tasks. Further studies are needed to demonstrate functional differences between TSS and ESS within the same individuals to effectively evaluate the advantages and disadvantages between these two modalities which may aid in choosing which strategy best fits a given individual's injury profile and goals. Based on the currently published data, the option to choose a modality will likely result in the most desirable patient-specific outcome.

Interestingly, when study participants were asked to voluntarily contract their lower limbs while stimulation was being delivered above motor threshold, the responses were inhibited (Figure 2). Furthermore, during joint-specific contractions, subjects inhibited all the recorded muscles bilaterally (Figure 3). Previous results using TSS in individuals without an SCI have indicated inhibition of responses during passive muscle stretching and muscle-tendon vibration, and facilitation of responses during voluntary muscle contraction [21,39]. Additionally, in previous TSS studies in individuals without an SCI, agonist lower-extremity muscle EMG responses were increased and antagonistic muscle responses were decreased while attempting voluntary movement $[24,28]$. Within our cohort of study participants with a severe $\mathrm{SCI}$, it is possible that post $\mathrm{SCI}$ reorganization in sensorimotor mapping has altered electrophysiological outputs resulting in simultaneous activation and reciprocal inhibition of agonist and antagonistic muscles during voluntary attempts at leg flexion and joint-specific movement [40]. Interestingly, individuals with chronic SCI typically exhibit increased excitability as evidenced by spasticity and hyperreflexia following the period of areflexia and spinal shock immediately following injury [41]. Therefore, current treatments to address spasticity include pharmacological agents that are used to reduce the excitability of the spinal cord, such as baclofen [42]. Physical treatments such as stretching, range of motion exercises, and voluntary contraction in individuals with incomplete SCI have shown improvements in spasticity, likely from enhanced activation of spinal inhibitory pathways [43]. Therefore, the present data align with the concept of increased inhibitory responses during physical tasks as well as data using TSS to attenuate spasticity in individuals with SCI, which was hypothesized to work through pre-synaptic and/or post-synaptic pathways [33]. It is noteworthy that previous results have shown bilateral facilitation of evoked responses during TSS when paired with transcranial magnetic stimulation (TMS) or galvanic vestibular stimulation (GVS), which activate the corticospinal and vestibulospinal tracts, respectively [44-47]. However, the present data suggest that stimulation of spinal cord circuitry combined with ongoing voluntary commands through remaining neural pathways crossing the lesion can inhibit spinally evoked motor responses.

Furthermore, when study participants were stratified according to the stimulation modality that was used and their injury severity as measured by their AIS classification, different patterns of evoked potential modulation emerged. AIS-A participants were able 
to inhibit responses across all measured muscles in ESS; however, AIS-A participants tested with TSS did not demonstrate similar results. Interestingly, participants who were classified as clinically incomplete (AIS-B/C) could inhibit the responses in at least 3 out of 4 recorded muscles (Figure 4). However, these results could not be shown to be statistically significant due to the low number of subjects in each subgroup. Previous studies have indicated that study participants with motor complete or incomplete injuries could regain voluntary motor function while using ESS [3]. Additionally, previous studies have indicated that healthy individuals [28,48] and individuals with SCI [49] could modulate TSS-evoked responses during functional tasks. However, in this study, we analyze the effect of voluntary effort on evoked response amplitude in participants with both clinically complete and incomplete SCI. These results suggest that individuals with less severe injury may be able to exert greater modulation on evoked responses recorded at motor threshold in the lower extremity. However, these findings are in a small cohort of participants and further work needs to be done to understand how remaining spinal cord fiber composition may affect lower-extremity function when paired with neuromodulation therapies. Recent mechanistic studies have suggested that the recovery of function following SCI can be attributed to propriospinal [50,51] and reorganization of cortico-reticulo-spinal tracts [52]. Additionally, motor-evoked responses and muscles activated can be modulated based on the timing that the pulse is delivered within a movement in humans and animals with SCI, which may contribute to the findings presented here as the subjects remained in the supine position continuously attempting flexion across multiple joints $[49,53]$. Therefore, future work should focus on the role of effort at different stages from preparation to execution of the movement and identifying the contributions of different spinal tracts to the recovery of function within the SCI population.

$\mathrm{SCI}$ is a heterogeneous population and results may differ depending on location and severity of injury, time since injury, and age of participant, therefore, further studies into the voluntary modulation of TSS- and ESS-evoked responses across clinical diagnoses are warranted. All of our experiments used low-frequency $(0.2-2 \mathrm{~Hz})$ stimulation in order to evaluate the effects of stimulation and voluntary effort without post-activation depression due to frequent stimulation. However, recent studies demonstrating return of function with spinal stimulation in individuals with severe paralysis have been at higher frequencies $[3,7,13,14]$, and the motor output during stimulation of the spinal networks at higher or lower frequency can be dramatically different, quantitatively and qualitatively [16]. It is plausible that during higher frequencies (e.g., above $25 \mathrm{~Hz}$ ) of spinal stimulation, the excitation predominates inhibition [54], which results in voluntary movements in the presence of spinal stimulation. Additionally, recent results using TSS have indicated that repeated exposure to stimulation may increase motoneuron output [55]. All participants within this study were not trained to perform the task, and therefore may exhibit different results when part of a long-term study. Additionally, the stimulation paradigm used within TSS for this study was composed of monophasic pulses, whereas ESS was delivered using biphasic pulses. Furthermore, the global motor threshold for this study was intentionally set at a low value of $20 \mu \mathrm{V}$; to observe supra-motor threshold responses of motor pools projecting to different muscles, which due to their multi-segmental origin are expected to have different thresholds. This low threshold value may have affected the ability of the subjects to modulate the responses, and further work should be performed to elucidate the effect of voluntary effort on spinally evoked responses at a range of different stimulation intensities. Lastly, the results we report were generated while study participants were positioned supine; however, body positioning influences recruitment of neural structures during spinal stimulation, and future work should evaluate the effect of voluntary intent during different body positions and tasks $[23,56]$.

\section{Conclusions}

In the present study, we found that individuals with severe SCI could modulate EMG outputs in their lower extremity, below their level of injury in the presence of spinal 
stimulation. During stimulation, both TSS and ESS pulses could elicit responses in lowerextremity musculature. Importantly, with low-frequency stimulation at motor threshold, both epidural and transcutaneous spinally evoked motor responses were inhibited, when participants voluntarily attempted to activate their lower-extremity muscles. However, study participants with clinically complete SCI using ESS and participants with clinically incomplete SCI using TSS demonstrated greater ability to modulate evoked responses than participants with clinically complete SCI using TSS. These results suggest the interaction of supraspinal and spinal mechanisms even in individuals with severe SCI.

Author Contributions: Conceptualization, J.S.C., P.J.G. and D.G.S.; methodology, J.S.C., M.L.G., M.B.L., D.D.V., A.R.T., C.L., I.A.L., P.J.G. and D.G.S.; validation, J.S.C., M.L.G., P.J.G. and D.G.S.; formal analysis, J.S.C., M.L.G., C.L., P.J.G. and D.G.S.; investigation, J.S.C., Y.P.G., V.R.E., I.A.L., K.D.Z., P.J.G. and D.G.S.; resources, K.H.L., K.D.Z. and D.G.S.; data curation, J.S.C., M.L.G., M.B.L., D.D.V., A.R.T., C.L., P.J.G. and D.G.S.; writing—original draft preparation, J.S.C. and D.G.S.; writing-review and editing, J.S.C., M.L.G., M.B.L., D.D.V., A.R.T., C.L., K.H.L., Y.P.G., V.R.E., I.A.L., K.D.Z., P.J.G. and D.G.S.; visualization, J.S.C., P.J.G. and D.G.S.; supervision, K.H.L., Y.P.G., V.R.E., I.A.L., K.D.Z., P.J.G. and D.G.S.; project administration, D.D.V., A.R.T., K.D.Z., P.J.G. and D.G.S.; funding acquisition, K.H.L., Y.P.G., V.R.E., K.D.Z., P.J.G. and D.G.S. All authors have read and agreed to the published version of the manuscript.

Funding: Sources of funding for the work reported here include the National Institutes of Health grant 1 R01 NS102920-01A1, The Grainger Foundation, Regenerative Medicine Minnesota, Jack Jablonski Bel13ve in Miracles Foundation, Mayo Clinic Graduate School of Biomedical Sciences, Mayo Clinic Center for Regenerative Medicine, Mayo Clinic Rehabilitation Medicine Research Center, Mayo Clinic Transform the Practice, Minnesota Office of Higher Education's Spinal Cord Injury and Traumatic Brain Injury Research Grant, Craig H. Neilsen Foundation, Dana and Albert R. Broccoli Charitable Foundation, Christopher and Dana Reeve Foundation, Walkabout Foundation, and Wings for Life Foundation.

Institutional Review Board Statement: The experimental procedures described herein were approved by the respective University of California, Los Angeles (UCLA) and Mayo Clinic institutional review boards.

Informed Consent Statement: Study participants provided written, informed consent to the experimental procedures.

Data Availability Statement: The data presented in this study are available on request from the corresponding author. The data are not publicly available due to potential patient privacy risks.

Acknowledgments: We thank the study participants for volunteering for this study. We also thank the Edgerton Neuromuscular Research Laboratory at UCLA, the Houston Methodist Neuromodulation \& Recovery Laboratory, and the Mayo Clinic Assistive and Restorative Technology Laboratory research support staff for their contributions to study design, data collection and general study support.

Conflicts of Interest: Y.P.G., researcher on the study team, holds shareholder interest in NeuroRecovery Technologies and Cosyma. He holds certain inventorship rights on intellectual property licensed by the regents of the University of California to NeuroRecovery Technologies and its subsidiaries. V.R.E., researcher on the study team holds shareholder interest in SpineX and NeuroRecovery Technologies and holds certain inventorship rights on intellectual property licensed by The Regents of the University of California to NeuroRecovery Technologies and its subsidiaries.

\section{References}

1. Cho, N.; Squair, J.W.; Bloch, J.; Courtine, G. Neurorestorative interventions involving bioelectronic implants after spinal cord injury. Bioelectron. Med. 2019, 5, 10. [CrossRef] [PubMed]

2. Megía García, A.; Serrano-Muñoz, D.; Taylor, J.; Avendaño-Coy, J.; Gómez-Soriano, J. Transcutaneous Spinal Cord Stimulation and Motor Rehabilitation in Spinal Cord Injury: A Systematic Review. Neurorehabil. Neural Repair 2020, 34, 3-12. [CrossRef] [PubMed]

3. Angeli, C.A.; Edgerton, V.R.; Gerasimenko, Y.P.; Harkema, S.J. Altering spinal cord excitability enables voluntary movements after chronic complete paralysis in humans. Brain 2014, 137, 1394-1409. [CrossRef] 
4. Calvert, J.S.; Grahn, P.J.; Strommen, J.A.; Lavrov, I.A.; Beck, L.A.; Gill, M.L.; Linde, M.B.; Brown, D.A.; Van Straaten, M.G.; Veith, D.D.; et al. Electrophysiological guidance of epidural electrode array implantation over the human lumbosacral spinal cord to enable motor function after chronic paralysis. J. Neurotrauma 2019, 36, 1451-1460. [CrossRef]

5. Darrow, D.; Balser, D.; Netoff, T.I.; Krassioukov, A.; Phillips, A.; Parr, A.; Samadani, U. Epidural Spinal Cord Stimulation Facilitates Immediate Restoration of Dormant Motor and Autonomic Supraspinal Pathways after Chronic Neurologically Complete Spinal Cord Injury. J. Neurotrauma 2019, 36, 2325-2336. [CrossRef] [PubMed]

6. Gerasimenko, Y.P.; Lu, D.C.; Modaber, M.; Zdunowski, S.; Gad, P.; Sayenko, D.G.; Morikawa, E.; Haakana, P.; Ferguson, A.R.; Roy, R.R.; et al. Noninvasive Reactivation of Motor Descending Control after Paralysis. J. Neurotrauma 2015, 32, 1968-1980. [CrossRef] [PubMed]

7. Grahn, P.J.; Lavrov, I.A.; Sayenko, D.G.; Van Straaten, M.G.; Gill, M.L.; Strommen, J.A.; Calvert, J.S.; Drubach, D.I.; Beck, L.A.; Linde, M.B.; et al. Enabling Task-Specific Volitional Motor Functions via Spinal Cord Neuromodulation in a Human With Paraplegia. Mayo Clin. Proc. 2017, 92, 544-554. [CrossRef] [PubMed]

8. Harkema, S.; Gerasimenko, Y.; Hodes, J.; Burdick, J.; Angeli, C.; Chen, Y.; Ferreira, C.; Willhite, A.; Rejc, E.; Grossman, R.G.; et al. Effect of epidural stimulation of the lumbosacral spinal cord on voluntary movement, standing, and assisted stepping after motor complete paraplegia: A case study. Lancet 2011, 377, 1938-1947. [CrossRef]

9. Rejc, E.; Angeli, C.; Harkema, S. Effects of Lumbosacral Spinal Cord Epidural Stimulation for Standing after Chronic Complete Paralysis in Humans. PLoS ONE 2015, 10, e0133998. [CrossRef]

10. Rejc, E.; Angeli, C.A.; Bryant, N.; Harkema, S.J. Effects of Stand and Step Training with Epidural Stimulation on Motor Function for Standing in Chronic Complete Paraplegics. J. Neurotrauma 2017, 34, 1787-1802. [CrossRef] [PubMed]

11. Rejc, E.; Angeli, C.A.; Atkinson, D.; Harkema, S.J. Motor recovery after activity-based training with spinal cord epidural stimulation in a chronic motor complete paraplegic. Sci. Rep. 2017, 7, 13476. [CrossRef] [PubMed]

12. Sayenko, D.; Rath, M.; Ferguson, A.R.; Burdick, J.; Havton, L.; Edgerton, V.R.; Gerasimenko, Y. Self-assisted standing enabled by non-invasive spinal stimulation after spinal cord injury. J. Neurotrauma 2018, 36, 1435-1450. [CrossRef] [PubMed]

13. Gill, M.L.; Grahn, P.J.; Calvert, J.S.; Linde, M.B.; Lavrov, I.A.; Strommen, J.A.; Beck, L.A.; Sayenko, D.G.; Van Straaten, M.G.; Drubach, D.I.; et al. Neuromodulation of lumbosacral spinal networks enables independent stepping after complete paraplegia. Nat. Med. 2018, 24, 1677-1682. [CrossRef] [PubMed]

14. Wagner, F.B.; Mignardot, J.; Le Goff-Mignardot, C.G.; Demesmaeker, R.; Komi, S.; Capogrosso, M.; Rowald, A.; Seáñez, I.; Caban, M.; Pirondini, E.; et al. Targeted neurotechnology restores walking in humans with spinal cord injury. Nature 2018, 563, 65-71. [CrossRef] [PubMed]

15. Angeli, C.A.; Boakye, M.; Morton, R.A.; Vogt, J.; Benton, K.; Chen, Y.; Ferreira, C.K.; Harkema, S.J. Recovery of Over-Ground Walking after Chronic Motor Complete Spinal Cord Injury. N. Engl. J. Med. 2018, 379, 1244-1250. [CrossRef] [PubMed]

16. Calvert, J.S.; Grahn, P.J.; Zhao, K.D.; Lee, K.H. Emergence of Epidural Electrical Stimulation to Facilitate Sensorimotor Network Functionality After Spinal Cord Injury. Neuromodulation 2019, 22, 244-252. [CrossRef]

17. Taccola, G.; Sayenko, D.; Gad, P.; Gerasimenko, Y.; Edgerton, V.R. And yet it moves: Recovery of volitional control after spinal cord injury. Prog. Neurobiol. 2018, 160, 64-81. [CrossRef] [PubMed]

18. Hofstoetter, U.S.; Freundl, B.; Binder, H.; Minassian, K. Common neural structures activated by epidural and transcutaneous lumbar spinal cord stimulation: Elicitation of posterior root-muscle reflexes. PLoS ONE 2018, 13, e0192013. [CrossRef] [PubMed]

19. Capogrosso, M.; Wenger, N.; Raspopovic, S.; Musienko, P.; Beauparlant, J.; Bassi Luciani, L.; Courtine, G.; Micera, S. A Computational Model for Epidural Electrical Stimulation of Spinal Sensorimotor Circuits. J. Neurosci. 2013, 33, 19326-19340. [CrossRef]

20. Ladenbauer, J.; Minassian, K.; Hofstoetter, U.S.; Dimitrijevic, M.R.; Rattay, F. Stimulation of the human lumbar spinal cord with implanted and surface electrodes: A computer simulation study. IEEE Trans. Neural Syst. Rehabil. Eng. 2010, 18, 637-645. [CrossRef]

21. Milosevic, M.; Masugi, Y.; Sasaki, A.; Sayenko, D.G.; Nakazawa, K. On the reflex mechanisms of cervical transcutaneous spinal cord stimulation in human subjects. J. Neurophysiol. 2019, 121, 1672-1679. [CrossRef]

22. Wu, Y.K.; Levine, J.M.; Wecht, J.R.; Maher, M.T.; LiMonta, J.M.; Saeed, S.; Santiago, T.M.; Bailey, E.; Kastuar, S.; Guber, K.S.; et al. Posteroanterior cervical transcutaneous spinal stimulation targets ventral and dorsal nerve roots. Clin. Neurophysiol. 2020, 131, 451-460. [CrossRef] [PubMed]

23. Danner, S.M.; Krenn, M.; Hofstoetter, U.S.; Toth, A.; Mayr, W.; Minassian, K. Body position influences which neural structures are recruited by lumbar transcutaneous spinal cord stimulation. PLOS ONE 2016, 11, e0147479. [CrossRef]

24. Minassian, K.; Persy, I.; Rattay, F.; Pinter, M.M.; Kern, H.; Dimitrijevic, M.R. Human lumbar cord circuitries can be activated by extrinsic tonic input to generate locomotor-like activity. Hum. Mov. Sci. 2007, 26, 275-295. [CrossRef] [PubMed]

25. Hofstoetter, U.S.; Freundl, B.; Binder, H.; Minassian, K. Recovery cycles of posterior root-muscle reflexes evoked by transcutaneous spinal cord stimulation and of the H reflex in individuals with intact and injured spinal cord. PLoS ONE 2019, 14, e0227057. [CrossRef] [PubMed]

26. Dimitrijevic, M.R.; Dimitrijevic, M.M.; Faganel, J.; Sherwood, A.M. Suprasegmentally induced motor unit activity in paralyzed muscles of patients with established spinal cord injury. Ann. Neurol. 1984, 16, 216-221. [CrossRef]

27. Moss, C.W.; Kilgore, K.L.; Peckham, P.H. A novel command signal for motor neuroprosthetic control. Neurorehabil. Neural Repair 2011, 25, 847-854. [CrossRef] [PubMed] 
28. Hofstoetter, U.S.; Minassian, K.; Hofer, C.; Mayr, W.; Rattay, F.; Dimitrijevic, M.R. Modification of reflex responses to lumbar posterior root stimulation by motor tasks in healthy subjects. Artif. Organs 2008, 32, 644-648. [CrossRef] [PubMed]

29. Minassian, K.; Persy, I.; Rattay, F.; Dimitrijevic, M.R.; Hofer, C.; Kern, H. Posterior root-muscle preflexes elicited by transcutaneous stimulation of the human lumbosacral cord. Muscle Nerve 2007, 35, 327-336. [CrossRef] [PubMed]

30. Gill, M.L.; Linde, M.B.; Hale, R.F.; Lopez, C.; Fautsch, K.J.; Calvert, J.S.; Veith, D.D.; Beck, L.A.; Garlanger, K.L.; Sayenko, D.G.; et al. Alterations of Spinal Epidural Stimulation-Enabled Stepping by Descending Intentional Motor Commands and Proprioceptive Inputs in Humans With Spinal Cord Injury. Front. Syst. Neurosci. 2020, 14, 590231. [CrossRef]

31. Gill, M.; Linde, M.; Fautsch, K.; Hale, R.; Lopez, C.; Veith, D.; Calvert, J.; Beck, L.; Garlanger, K.; Edgerton, R.; et al. Epidural Electrical Stimulation of the Lumbosacral Spinal Cord Improves Trunk Stability During Seated Reaching in Two Humans With Severe Thoracic Spinal Cord Injury. Front. Syst. Neurosci. 2020, 14, 79. [CrossRef]

32. Rath, M.; Vette, A.H.; Ramasubramaniam, S.; Li, K.; Burdick, J.; Edgerton, V.R.; Gerasimenko, Y.P.; Sayenko, D.G. Trunk Stability Enabled by Noninvasive Spinal Electrical Stimulation after Spinal Cord Injury. J. Neurotrauma 2018, 35, 2540-2553. [CrossRef] [PubMed]

33. Hofstoetter, U.S.; Freundl, B.; Danner, S.M.; Krenn, M.J.; Mayr, W.; Binder, H.; Minassian, K. Transcutaneous Spinal Cord Stimulation Induces Temporary Attenuation of Spasticity in Individuals with Spinal Cord Injury. J. Neurotrauma 2020, 37, 481-493. [CrossRef] [PubMed]

34. Calvert, J.S.; Manson, G.A.; Grahn, P.J.; Sayenko, D.G. Preferential activation of spinal sensorimotor networks via lateralized transcutaneous spinal stimulation in neurologically intact humans. J. Neurophysiol. 2019, 122, 2111-2118. [CrossRef] [PubMed]

35. Krenn, M.; Toth, A.; Danner, S.M.; Hofstoetter, U.S.; Minassian, K.; Mayr, W. Selectivity of transcutaneous stimulation of lumbar posterior roots at different spinal levels in humans. Biomed. Tech. 2013, 58 (Suppl. 1), 2-3. [CrossRef]

36. Sayenko, D.G.; Angeli, C.; Harkema, S.J.; Edgerton, V.R.; Gerasimenko, Y.P. Neuromodulation of evoked muscle potentials induced by epidural spinal-cord stimulation in paralyzed individuals. J. Neurophysiol. 2014, 111, 1088-1099. [CrossRef] [PubMed]

37. Sayenko, D.G.; Atkinson, D.A.; Dy, C.J.; Gurley, K.M.; Smith, V.L.; Angeli, C.; Harkema, S.J.; Edgerton, V.R.; Gerasimenko, Y.P. Spinal segment-specific transcutaneous stimulation differentially shapes activation pattern among motor pools in humans. $J$. Appl. Physiol. 2015, 118, 1364-1374. [CrossRef]

38. Cuellar, C.A.; Mendez, A.A.; Islam, R.; Calvert, J.S.; Grahn, P.J.; Knudsen, B.; Pham, T.; Lee, K.H.; Lavrov, I.A. The Role of Functional Neuroanatomy of the Lumbar Spinal Cord in Effect of Epidural Stimulation. Front. Neuroanat. 2017, 11, 82. [CrossRef]

39. Kato, T.; Sasaki, A.; Yokoyama, H.; Milosevic, M.; Nakazawa, K. Effects of neuromuscular electrical stimulation and voluntary commands on the spinal reflex excitability of remote limb muscles. Exp. Brain Res. 2019, 237, 3195-3205. [CrossRef]

40. Crone, C.; Johnsen, L.L.; Biering-Sørensen, F.; Nielsen, J.B. Appearance of reciprocal facilitation of ankle extensors from ankle flexors in patients with stroke or spinal cord injury. Brain 2003, 126, 495-507. [CrossRef]

41. Dietz, V. Behavior of spinal neurons deprived of supraspinal input. Nat. Rev. Neurol. 2010, 6, 167-174. [CrossRef]

42. Dario, A.; Tomei, G. A benefit-risk assessment of baclofen in severe spinal spasticity. Drug Saf. 2004, 27, 799-818. [CrossRef]

43. D'Amico, J.M.; Condliffe, E.G.; Martins, K.J.B.; Bennett, D.J.; Gorassini, M.A. Recovery of neuronal and network excitability after spinal cord injury and implications for spasticity. Front. Integr. Neurosci. 2014, 8, 36. [CrossRef]

44. Andrews, J.C.; Stein, R.B.; Roy, F.D. Reduced postactivation depression of soleus H reflex and root evoked potential after transcranial magnetic stimulation. J. Neurophysiol. 2015, 114, 485-492. [CrossRef] [PubMed]

45. Knikou, M. Transpinal and transcortical stimulation alter corticospinal excitability and increase spinal output. PLoS ONE 2014, 9, e102313. [CrossRef] [PubMed]

46. Roy, F.D.; Bosgra, D.; Stein, R.B. Interaction of transcutaneous spinal stimulation and transcranial magnetic stimulation in human leg muscles. Exp. Brain Res. 2014, 232, 1717-1728. [CrossRef] [PubMed]

47. Sayenko, D.; Atkinson, D.; Mink, A.; Gurley, K.; Edgerton, R.; Harkema, S.J.; Gerasimenko, Y. Vestibulospinal and corticospinal modulation of lumbosacral network excitability in human subjects. Front. Physiol. 2018, 9, 1746. [CrossRef]

48. Courtine, G.; Harkema, S.J.; Dy, C.J.; Gerasimenko, Y.P.; Dyhre-Poulsen, P. Modulation of multisegmental monosynaptic responses in a variety of leg muscles during walking and running in humans. J. Physiol. 2007, 582, 1125-1139. [CrossRef]

49. Dy, C.J.; Gerasimenko, Y.P.; Edgerton, V.R.; Dyhre-Poulsen, P.; Courtine, G.; Harkema, S.J. Phase-Dependent Modulation of Percutaneously Elicited Multisegmental Muscle Responses After Spinal Cord Injury. J. Neurophysiol. 2010, 103, $2808-2820$. [CrossRef]

50. Courtine, G.; Song, B.; Roy, R.R.; Zhong, H.; Herrmann, J.E.; Ao, Y.; Qi, J.; Edgerton, V.R.; Sofroniew, M.V. Recovery of supraspinal control of stepping via indirect propriospinal relay connections after spinal cord injury. Nat. Med. 2008, 14, 69-74. [CrossRef]

51. Formento, E.; Minassian, K.; Wagner, F.; Mignardot, J.B.; Le Goff-Mignardot, C.G.; Rowald, A.; Bloch, J.; Micera, S.; Capogrosso, M.; Courtine, G. Electrical spinal cord stimulation must preserve proprioception to enable locomotion in humans with spinal cord injury. Nat. Neurosci. 2018, 21, 1728-1741. [CrossRef]

52. Asboth, L.; Friedli, L.; Beauparlant, J.; Martinez-Gonzalez, C.; Anil, S.; Rey, E.; Baud, L.; Pidpruzhnykova, G.; Anderson, M.A.; Shkorbatova, P.; et al. Cortico-reticulo-spinal circuit reorganization enables functional recovery after severe spinal cord contusion. Nat. Neurosci. 2018, 21, 576-588. [CrossRef] [PubMed]

53. Lavrov, I.; Dy, C.J.; Fong, A.J.; Gerasimenko, Y.; Courtine, G.; Zhong, H.; Roy, R.R.; Edgerton, V.R. Epidural Stimulation Induced Modulation of Spinal Locomotor Networks in Adult Spinal Rats. J. Neurosci. 2008, 28, 6022-6029. [CrossRef] 
54. Hofstoetter, U.S.; Danner, S.M.; Freundl, B.; Binder, H.; Mayr, W.; Rattay, F.; Minassian, K. Periodic modulation of repetitively elicited monosynaptic reflexes of the human lumbosacral spinal cord. J. Neurophysiol. 2015, 114, 400-410. [CrossRef] [PubMed]

55. Murray, L.M.; Knikou, M. Transspinal stimulation increases motoneuron output of multiple segments in human spinal cord injury. PLoS ONE 2019, 14, e213696. [CrossRef]

56. Militskova, A.; Mukhametova, E.; Fatykhova, E.; Sharifullin, S.; Cuellar, C.A.; Calvert, J.S.; Grahn, P.J.; Baltina, T.; Lavrov, I. Supraspinal and Afferent Signaling Facilitate Spinal Sensorimotor Network Excitability After Discomplete Spinal Cord Injury: A Case Report. Front. Neurosci. 2020, 14, 552. [CrossRef] [PubMed] 